\title{
Severe lactic acidosis from acute cyanide poisoning after intentional amygdalin ingestion in a teenager
}

\author{
Jack Green \\ Division of Pediatric Critical Care Medicine, Cedars-Sinai Medical Center, Los Angeles, CA, USA
}

\begin{abstract}
Cyanide poisoning via the oral route is a remarkably rare entity in the United States. Though acute toxicity from this poison may present with classic signs and symptoms (smell of bitter almonds on breath and cherry-red skin), these signs are frequently not clinically observed in the intoxicated patient, making it low on the routine differential diagnosis leading to both diagnostic and therapeutic challenges for the bedside clinician. This is a case of a 17-yearold male with a history of depression who presented to the Emergency Room (ER) with altered mental status, abdominal pain, and emesis. A severely elevated and worrisome lactic acidosis triggered the ER's septic shock bundle and algorithm, but further investigation ultimately led to the unifying diagnosis of intentional cyanide poisoning.
\end{abstract}

\section{Case Report}

The patient is a 17-year-old male with a history of depression who was in his usual state of health until he was found with altered sensorium, obtunded, and hypotonic in a bathtub by his sibling. The patient's unresponsiveness prompted the sibling to bring him in to the ER. The history was initially difficult to obtain, but the

Correspondence: Jack Green, Division of Pediatric Critical Care Medicine, Cedars-Sinai Medical Center, 8700 Beverly Blvd, NT 4230, Los Angeles, CA 90048, USA

Tel.: 001.310.423.8080

E-mail: jack.green@cshs.org

Key words: cyanide; suicidality; pediatrics.

Conflict of interest: No one. This work was not supported by any grant.

Ethics approval and consent to participate: The manuscript does not contain any elements that would allow the recognition of the patient.

Received for publication: 26 March 2020.

Accepted for publication: 22 August 2020.

This work is licensed under a Creative Commons Attribution 4.0 License (by-nc 4.0).

(C) Copyright: the Author(s), 2020

Licensee PAGEPress, Italy

Emergency Care Journal 2020; 16:8985

doi:10.4081/ecj.2020.8985 patient later endorsed abdominal pain over the prior weeks prompting an Over-The-Counter (OTC) medication that he selfadministered approximately nine hours prior to arrival in the ER. Several hours after administration, he woke up in the middle of the night with even worse pain, began to vomit, got into the shower, and subsequently lost consciousness.

In the ER, initial vital signs were an oral temperature of $36.3 \mathrm{C}$, heart rate of 127 beats per minute, blood pressure of $107 / 46$ $\mathrm{mmHg}$, respiratory rate of 18 breaths per minute, and an oxygen saturation of $100 \%$ on room air. Physical exam was notable for waxing/waning mental status, abdominal tenderness on palpation, dry mucous membranes, and mydriasis. There were no tremors or hypertonia. Laboratory findings revealed significant metabolic acidosis (AG $32 \mathrm{mEq} / \mathrm{L}$, serum bicarbonate $8 \mathrm{mmol} / \mathrm{L}$, arterial $\mathrm{pH}$ 7.24), severe hyperlactatemia $(15.1 \mathrm{mmol} / \mathrm{L}$, institutional range $0.5-2.2$ ), leukocytosis with neutrophilic predominance (WBC 21.95 1000/UL, 81.4\% PMNs), an elevated serum creatinine (1.31 $\mathrm{mg} / \mathrm{dL}$, previous baseline $0.7-0.9)$, and an arterial-to-venous oxygen partial pressure difference of 23 (paO2 $94 \mathrm{mmHg}$, pvO2 71 $\mathrm{mmHg}$ ). Urine and serum toxicology were normal (see Table 1 for expanded laboratory results). After resuscitation with volume expansion and oxygen support, along with an initial working diagnosis of sepsis, the patient began to arouse and was able to answer questions. Further psycho-social history was elicited, with admission to a history of depression on anti-depressant therapies. As the sepsis algorithm was carried out, he confessed to buying Amygdalin on the internet with no intention of self-harm bur rather aimed at abdominal pain symptom relief. This immediately triggered a call to local poison control, who recommended 5 grams of Intra-Venous (IV) hydroxycobalamin as the antidote administration for presumptive cyanide poisoning. Co-ingestions with methanol, ethylene glycol, iron, carbon monoxide, amphetamines, metformin, and caustic agents were considered but after admission of amygdalin ingestion and a routine negative toxicology screen, the aforementioned agents were not further investigated. The patient was then transferred to the Intensive Care Unit (ICU) for ongoing medical stabilization.

In the ICU, the patient's lactic acidosis improved within hours of hydroxycobalamin administration and he did not require further re-dosing. A discussion was had with local Poison Control about other antidotes but a decision was to continue close monitoring in light of the patient's improvement. A cyanide level was obtained from the blood and not surprisingly found to be in the undetectable range, particularly as the sample was drawn three hours after antidote administration. The patient was also pan-cultured and empiric antibiotics were quickly administered. One of the blood cultures grew coagulase-negative Staphylococcus which was deemed to be a contaminant, with subsequent sterile blood cultures. In the ICU, arterial and venous blood gases were closely monitored, intravenous fluids and supportive therapies were continued, and within twelve hours his lactate levels normalized. After frequent laboratory checks and neurologic assessments, he was stable for transfer 
from the ICU to the general wards after 48 hours. There, he continued to be observed with psychiatry consultants closely following with re-initiation of outpatient anti-depressants. After being medically cleared, he was then transferred to an inpatient psychiatric facility. Though the patient initially denied the intentionality of this overdose, it was later discovered that he specifically sought ways to commit suicide on-line and was found in his browser search history.

\section{Discussion}

Acute cyanide poisoning remains relatively uncommon in the United States, especially in the pediatric population. Unless there is a clear history of smoke inhalation, generally prompting the practitioner to rule out toxicity from its gaseous form, recognition of its manifestations from solid or liquid sources remains a bedside challenge. Interestingly, several meta-analyses found that the vast majority of cyanide toxicity cases are caused by ingested forms of the toxin. ${ }^{1}$ Though uncommon in the United States, case reports in more tropical countries exist in pediatrics regarding oral cyanide toxicity. Among those, several describe ingestion of apricot kernels, bitter almonds, and cassava plant leading to similar symptomatology of vomiting and altered mental status. ${ }^{2-4}$ All cases required detailed investigations of food and drug histories or high levels of clinical suspicion leading to the diagnosis. In our patient, admission to the ingestion of an OTC powdered supplement called Amygdalin aided in confirmation of the diagnosis. Though he purported taking the supplement with the belief it would help his recent abdominal pain, taking into account the psycho-social history, intentional ingestion with an underlying knowledge of the

Table 1. Initial laboratory testing for a teenager presenting with emesis and altered mental status.

\begin{tabular}{|c|c|c|}
\hline Lab Test & Result & Reference or therapeutic ranges \\
\hline White Blood Cell Count (1000/UL) & 21.95 & $4.00-11.00$ \\
\hline Hemoglobin ( $\mathrm{g} / \mathrm{dL})$ & 17.8 & $13.0-17.0$ \\
\hline Hematocrit (\%) & 54.0 & $37.5-49.9$ \\
\hline Platelets (1000/UL) & 249 & $150-450$ \\
\hline Neutrophils (\%) & 81.4 & $\mathrm{~N} / \mathrm{A}$ \\
\hline Lymphocytes (\%) & 4.8 & $\mathrm{~N} / \mathrm{A}$ \\
\hline Eosinophils (\%) & 1.5 & $\mathrm{~N} / \mathrm{A}$ \\
\hline pH-arterial & 7.24 & $7.35-7.45$ \\
\hline pCO2- arterial (mmHg) & 36 & $35-45$ \\
\hline pO2- arterial (mmHg) & 164 & $75-100$ \\
\hline HCO3- arterial (mmol/L) & 15.4 & $23-29$ \\
\hline Base Excess- arterial (mmol/L) & -12 & -2 to +2 \\
\hline Lactate - arterial (mmol/L) & 15.1 & $0.5-2.2$ \\
\hline Glucose (mg/dL) & 125 & $70-99$ \\
\hline Sodium $(\mathrm{mmol} / \mathrm{L})$ & 144 & $135-145$ \\
\hline Potassium (mmol/L) & 3.4 & $3.5-5.0$ \\
\hline Chloride (mmol/L) & 107 & $98-107$ \\
\hline Carbon Dioxide (mmol/L) & 8 & $22-31$ \\
\hline Anion Gap (mEq/L) & 32 & $10-20$ \\
\hline Urea Nitrogen (mg/dL) & 13.8 & $8.4-21.0$ \\
\hline Creatinine (mg/dL) & 1.31 & $0.69-1.1$ \\
\hline Albumin $(\mathrm{g} / \mathrm{dL})$ & 5.4 & $4.1-5.1$ \\
\hline ALT (U/L) & 27 & $0-55$ \\
\hline AST (U/L) & 54 & $5-34$ \\
\hline Lipase (U/L) & 18 & $8-78$ \\
\hline TSH (mIU/L) & 0.54 & $0.39-4.60$ \\
\hline Creatine Kinase (U/L) & 344 & $30-200$ \\
\hline Urine cocaine metabolites & NEG & NEG \\
\hline Urine methadone & NEG & NEG \\
\hline Urine opiate & NEG & NEG \\
\hline Urine phencyclidine & NEG & NEG \\
\hline Serum acetaminophen (mg/L) & $<1$ & $10-30$ \\
\hline Serum ethanol (g/dL) & None detected & None detected \\
\hline Serum salicylate (mg/L) & $<50$ & $150-300$ \\
\hline Serum osmolality (mOSM/kg) & 310 & $275-295$ \\
\hline
\end{tabular}


drug's toxic metabolites was deemed more likely.

A patient who presents with tachycardia, wide pulse pressure, and altered sensorium with primary lab findings of leukocytosis and lactic acidosis immediately triggered a rule out sepsis alarm in the ED. However, a very broad differential in the setting of an initially limited history is important so as not to exclude other possible diagnoses. In our patient, these may include abdominal pathology such as peritonitis or pancreatitis, new onset Diabetic KetoAcidosis (DKA), thyroid storm, drug-drug interactions leading to Serotonin Syndrome (SS) or Neuroleptic Malignant Syndrome (NMS), accidental toxic environmental exposure, and intentional drug ingestion. Subtle clues make sepsis, the ER's top initial differential, less likely. There was no history of fever, antecedent illnesses, sick contacts, or pertinent medical co-morbidities to suggest sepsis. The absence of tremors, hypertonia, and pyrexia easily excluded SS and NMS. Lipase and amylase were normal excluding abdominal pathology. A normal blood glucose excluded classis DKA. Thyroid levels were normal excluding thyroid storm. This leaves us with toxic exposure of some kind. Co-ingestions with methanol, ethylene glycol, iron, carbon monoxide, amphetamines, metformin, and caustic agents were part of the differential diagnosis for metabolic acidosis (Table 2). Gas chromatography massspectroscopy would be useful in future clinical scenarios to rule out more occult ingestions. Indeed, clinically significant altered sensorium and emesis occurred in fact only after OTC administration. And after further investigation, the patient finally endorsed a history of depression on anti-depressant therapies and admits to consuming a homeopathic supplement called Amygdalin. A cyanogenic glycoside, ${ }^{5}$ in excess this drug is known to lead to acute cyanide poisoning, the unifying diagnosis in our patient.

Amygdalin - a cyanogenic glycoside - is hydrolyzed in the gut to hydrogen cyanide, glucose, benzaldehyde, and beta-glucosidase emulsin which further perpetuates the breakdown of amygdalin leading to ongoing cyanide release into the bloodstream. ${ }^{6}$ Once in the blood, inhibition of key enzymes associated with the mitochondrial electron transport chain causes significant down-regulation of

Table 2. Approach to differential diagnoses for Metabolic Acidosis (MA) in the pediatric population. ${ }^{13}$

\begin{tabular}{lc}
\hline Non-anion gap MA & Anion-gap MA \\
Gastroenteritis & Diabetic ketoacidosis \\
Renal tubular acidosis & Lactic acidosis due to: \\
Septic shock \\
Cardiogenic shock \\
Renal failure \\
Toxic ingestions due to: \\
Ethylene glycol \\
Ethanol \\
Methanol \\
Propylene glycol \\
Isopropanol \\
Salicylates \\
Iron \\
Isoniazid \\
Metformin \\
Carbon monoxide \\
Toluene \\
Cyanide \\
Inborn errors of metabolism
\end{tabular}

aerobic metabolism necessary to sustain normal cellular function. This ultimately leads to profound Type-B lactic acidosis. As opposed to Type-A lactic acidosis, generally caused by a hypoxemic or hypo-perfused state such as in septic shock, hyperlactatemia in our patient was caused by the mitochondria's inability to adequately sustain aerobic respiration. ${ }^{7}$ Unfortunately, the symptoms that manifest after cyanide ingestion can be very generalized and non-specific making the diagnosis quite challenging without a proper history. Our patient met other lab criteria that continued to support the diagnosis of cyanide poisoning. In addition to a high anion-gap lactic acidosis, his labs also showed a very narrow arterial-to-venous oxygen content gradient as evidenced by his first concurrent arterial and venous oxygen levels $(94 \mathrm{mmHg}$ and 71 $\mathrm{mmHg}$ respectively). His high venous oxygen levels further confirm his mitochondria's inability to appropriately extract and utilize oxygen.

After the ER confidently confirmed the diagnosis, the local poison control center was contacted with recommendations to immediately administer hydroxycobalamin as the monotherapy antidote. Hydroxycobalamin is a direct binder of cyanide, forming cyanocobalamin or Vitamin B12 which is then renally excreted. ${ }^{8}$ Other antidotes for cyanide poisoning exist and include amyl nitrite, sodium nitrite, and sodium thiosulfate, otherwise known as CyanoKit. These antidotes combined act by de-toxifying cyanide into the form of thiocyanate which is then excreted renally. ${ }^{9}$ Since a byproduct of this mechanism is methemoglobinemia, methylene blue is sometimes co-administered to negate those effects. Additionally, our patient presented to the ER more than twelve hours after ingestion. After a thorough discussion with local Poison Control, gastric decontamination was also not implemented due to this timeline. However, it would have been entirely reasonable due to the lethality of cyanide at low doses. ${ }^{10}$ Because data now suggests that hydroxycobalamin is safe and effective in pediatrics, it became the antidote of choice in our patient with rapid improvement thereafter.

The Centers for Disease Control and Prevention (CDC) estimates that roughly $14 \%$ of high school students make a suicide plan, of which approximately half carry out said plan. ${ }^{11}$ These numbers are increasing over the last decade, and unfortunately remain the second leading cause of death in teenagers. While varying modalities for suicide differ depending on age, gender and other factors, suicide by self-poisoning is still the most prevalent in this vulnerable age group. In fact, nearly 1.7 million cases of intentional self-poisoning have been reported over the last two decades. Of these, the National Poison Data System (NPDS) reports that Selective Serotonin Reuptake Inhibitors (SSRIs), Non-Steroidal Anti-Inflammatory Drugs (NSAIDs), acetaminophen, and anti-histamines are the most common substance categories, underscoring that a history of anti-depressant use markedly increases an individual's risk for suicide or subsequent suicide attempts. ${ }^{12}$ Our patient was later determined to have a previous history of suicidal ideation.

\section{Conclusion}

Acute cyanide poisoning remains a diagnostic challenge and requires a high index of clinical suspicion, especially with at-risk patient populations. Significant lactic acidosis not congruent with a classic presentation of septic shock should prompt investigation into toxic ingestions. 


\section{References}

1. Hendry-Hofer TB, Ng PC, Witeof AE, et al. A review on ingested cyanide: risks, clinical presentation, diagnostics, and treatment challenges. J Med Toxicol 2019;15:128-33.

2. Ekinci F, Yildizdas D, Ates A, et al. Cyanide intoxication by apricot kernels: a case report and literature review. Emerg Care J 2019;15:75-8.

3. Shragg TA, Albertson TE, Fisher CJ. Cyanide poisoning after bitter almond ingestion. West J Med 1982;136:65-9.

4. Ruangkanchanasetr S, Wananukul V, Suwanjutha, S. Cyanide poisoning, 2 cases report and treatment review. J Med Assoc Thai 1999;82:162-7.

5. Cressey P, Reeve J. Metabolism of cyanogenic glycosides: a review. Food Chem Toxicol 2019;125:225-32.

6. Geller RJ, Barthold C, Saiers JA, et al. Pediatric cyanide poisoning: causes, manifestations, management, and unmet needs. Pediatr 2006;118:2147-58.

. Baud FJ, Haidar MK, Jouffroy R, et al. Determinants of lactic acidosis in acute cyanide poisonings. Crit Care Med 2018;46:523-9.

8. Borron SW, Baud FJ, Megarbane B, et al. Hydroxocobalamin for severe acute cyanide poisoning by ingestion or inhalation. Amer J Emerg Med 2007;25:551-8.

9. Gracia R, Shepherd G. Cyanide poisoning and its treatment. Pharmacother 2004;24:1358 $\square 65$.

10. Cummings TF. The treatment of cyanide poisoning. Occup Med (Lond) 2004;54:82 $\square 5$.

11. Kann L, McManus T, Harris WA, et al. Youth risk behavior surveillance - United States, 2017. MMWR Surveill Summ 2018;67:100-14.

12. Gummin DD, Mowry JB, Spyker DA, et al. 2018 Annual report of the American Association of Poison Control Centers' National Poison Data System (NPDS): 36th annual report. Clin Toxicol 2019;57:1220-1413.

13. Kappy MS, Morrow G. A diagnostic approach to metabolic acidosis in children. Pediatr 1980;65:351-6. 